\title{
Psychological specifics of the professional activity of the subject-information class
}

\section{A. V. Karpov ${ }^{1}$, A. V. Chemyakina ${ }^{1}$}

${ }^{1}$ P. G. Demidov Yaroslavl State University, 14 Sovetskaya str., Yaroslavl 150003, Russian Federation

DOI: $10.18255 / 1996-5648-2021-3-422-433$

Research article Full text in Russian

The article presents theoretical and methodological materials that justify the need to differentiate a new class of professional activity - subjectinformation. This class is qualitatively specific in relation to two other traditionally distinguished classes: subject-object and subject-subject. Based on the material of the most representative types of activity of this class, which are implemented through computer technologies, its psychological characteristics are given. It is shown how the research of this class can contribute to the further development of the psychological theory of activity. A new approach to the research of this class of activity is formulated, based on the synthesis of the main provisions of the psychological theory of activity and modern metacognitivism.

Keywords: activity; types of activity; classes of activity; subject-object class; subject-subject class; subject-information class; activity structure; metacognitive processes

\section{INFORMATION ABOUT AUTHORS}

\author{
Karpov, Anatoly V. E-mail: anvikar56@yandex.ru \\ Corresponding Member of the RAO, Doc. Sc. \\ (Psychology), Professor
}

\begin{tabular}{l|l} 
Chemyakina, Anna V. & $\begin{array}{l}\text { E-mail: anyachemy@mail.ru } \\
\text { Cand. Sc. (Psychology), Associate Professor }\end{array}$
\end{tabular}

Funding: RSF, project 21-18-00039. 


\section{Психологическая специфика профессиональной деятельности субъектно-информационного класса}

А. В. Карпов ${ }^{1}$, А. В. Чемякина ${ }^{1}$

${ }^{1}$ Ярославский государственный университет им. П. Г. Демидова, ул. Советская, 14, Ярославль, 150003, Российская Федерация

DOI: $10.18255 / 1996-5648-2021-3-422-433$

УдК 159.9

Научная статья

Полный текст на русском языке

Представлены теоретические и методологические материалы, обосновывающие необходимость дифференциации нового класса профессиональной деятельности - субъектно-инфрормационного. Он является качественно специфическим по отношению к двум иным, традиционно выделяемым классам, - субъектно-объектному и субъектно-субъектному. На материале наиболее репрезентативных разновидностей деятельностей этого класса, которые реализуются посредством компьютерных технологий, дана его психологическая характеристика. Показано, каким образом исследование этого класса может содействовать дальнейшему развитию психологической теории деятельности. Сфрормулирован новый подход к изучению данного класса деятельности, базирующийся на синтезе основных положений психологической теории деятельности и современного метакогнитивизма.

Ключевые слова: деятельность; виды деятельности; классы деятельности; субъектно-объектный класс; субъектно-субъектный класс; субъектноинорормационный класс; структура деятельности; метакогнитивные процессы

\section{ИНФОРМАЦИЯ ОБ АВТОРАХ}

\begin{tabular}{c|l} 
Карпов, Анатолий Викторович & $\begin{array}{l}\text { E-mail: anvikar56@yandex.ru } \\
\text { Член-корреспондент PAO, доктор } \\
\text { психологических наук, профессор }\end{array}$ \\
Чемякина, Анна Вадимовна & $\begin{array}{l}\text { E-таil: anyachemy@mail.ru } \\
\text { Кандидат психологических наук, доцент }\end{array}$
\end{tabular}

Финансирование: РНФ, проект № 21-18-00039.

Одной из основных черт социо-экономического развития общества является объективно развертывающийся процесс эволюции и закономерной трансформации форм и видов, типов и классов профрессиональной деятельности - то, что обычно обозначается понятием «филогенеза дея(C) Карпов А. В., Чемякина А. В., 2021

Статья открытого доступа под лицензией CC BY (https://creativecommons.org/licenses/by/4.0/) 
Карпов А. В., Чемякина А. В.

тельности» [1-3]. В этом плане показательным является переход от доминирования в общественном разделении труда субъект-объектных видов деятельности к субъект-субъектнъм видам, а также смена их роли и места в нем [3]. В состав второго класса входят такие играющие действительно определяющую роль в современном обществе виды профрессиональной деятельности, как управленческая и организационная деятельность, образовательная деятельность во всех ее многочисленных разновидностях, врачебная деятельность, весь спектр различных видов сервисной деятельности и мн. др. [4-5]. Смена двух традиционно дифференцируемых классов, а также постепенное и неуклонное изменение приоритетов между ними в структуре общественного разделения труда - это и есть объективная по природе и магистральная по масштабу тенденция изменения мира профрессий. Важно то, что ограничиваться такой логикой - значит приуменьшать реальную сложность эволюции форм трудовой активности, ограничивать диапазон их прогресса и фрактически во многом закрывать возможность продуктивного и углубленного исследования все новых ее типов и разновидностей, а возможно, и классов. К нему принадлежат, как показано в частности в [1-2], многочисленные разновидности становящегося все более распространенным субъектно-инбормаиионного класса. Важно и то, что именно ему принадлежит будущее; это ставит вопрос о его приоритетном изучении, а также о синтезе представлений о нем и о разработке обобщающей психологической теории деятельности. Она должна синтезировать в себе представления как о субъект-объектных и субъект-субъектных ее типах, так и о ее субъектно-информационном типе.

Очень показательно, что именно это обстоятельство находит все более зримое и многоплановое подтверждение в ряде современных подходов к проблематике профессиональной деятельности. В частности, оно выступило одним из основных в целом ряде выполненных нами исследований (см., например, $[2,6])$. Было обосновано положение, согласно которому существующая дифрференциация огромного многообразия деятельностей («мира деятельностей») всего на два класса является упрощенной и недопустимо симплифицированной; она не отражает всего их реального многообразия ${ }^{1}$. «Мир деятельностей» динамичен, а мера этой динамичности, постоянно возрастая, в настоящее время достаточно велика. Она характеризуется перманентным возникновением принципиально новых видов деятельности и способов ее организации, не говоря уже о еще более быстром прогрессе их «технологической составляющей».

В связи с этим, как показывает специальный анализ всех отмеченных вопросов, и возникает необходимость дифференциации качественно специфического и несводимого к двум уже выделенным классам деятель-

${ }^{1}$ В этом плане модно привести выражение В. П. Зинченко, отмечавшего, что «деятельность - это такая беспредельность, которая вряд ли поддается какой-либо систематизации» [9]. 
ности - субъектно-информационного [7]. Его важнейшей отличительной характеристикой является то, что в нем имеет место та же самая в принципе трансформация (то есть трансформация, принципиальная по смыслу и радикальная по масштабу), которая привела в свое время к необходимости дифференциации субъект-объектного и субъект-субъектного классов. Это трансорормация основного атрибута деятельности - ее предмета. В субъектно-инфромационных видах деятельности им выступает уже не объект, но и не субъект, а совершенно иная и предельно специфическая сущность - инфбормация. Она сама по себе, то есть исходно, не является ни объектом, ни субъектом, хотя может сигнифицировать и тот и другой и по отдельности и одновременно. Сфрера действия и область представленности этого третьего класса предельно широка (см. далее); в его деятельности основной атрибут - предмет не только качественно трансформируется, но и еще более усложняется [8-10]. Причем такое усложнение происходит в самом прямом смысле данного понятия, поскольку деятельность данного класса становится еще более опосредствованной, а ее предмет - еще более имплицитным, вообще приобретая в ряде случаев черты именно качественно новой реальности - виртуальной.

Таким образом, со всей очевидностью и, более того, с объективной необходимостью фрормулируется задача приоритетного исследования именно этого пока не вполне традиционного, но крайне важного класса профессиональной деятельности. Уже выполненные к настоящему времени исследования вскрывают глубокую психологическую специфичность данного класса, наличие у него качественно иных по сравнению с двумя другим классами особенностей и закономерностей. Кроме того, как отмечалось выше, не менее значимо, что именно этому классу принадлежит будущее; он с очевидностью находится на «острие» прогресса видов и типов профессиональной деятельности - прогресса, масштабы и темпы которого не только велики, но и зачастую даже непредсказуемы. Он и его дальнейшая эволюция составляет не только ближайшую, но и отдаленную перспективу развития профессиональной деятельности, причем выраженную настолько, что в ряде случаев представления о ней вообще сводятся к постепенному вытеснению всех иных разновидностей профессиональной деятельности этим классом. Итак, значимость диффреренциации данного класса - и как объективно представленного, и как гносеологически дифрференцируемого (в качестве еще одной важнейшей экспликации общего предмета психологии профессиональной деятельности) - обусловлена тем, что он представляет собой несомненную и широко представленную реальность. С ней не только необходимо считаться, но именно ее и следует сделать предметом приоритетных исследований. Более того, такая смена приоритетов будет становиться все более явной по мере развития мира деятельности и изменения структуры общественного разделения труда. 
На первый план выходит важная в теоретическом плане задача формулировки такого методологического подхода, который был бы адекватен психологической природе данного класса деятельности, а также конструктивнен в плане его исследования. Такой подход был разработан нами [8]; его основная черта состоит в том, что он позволяет реализовать по отношению к исследованию данного класса основные положения метакогнитивизма.

Следует принимать во внимание то важнейшее обстоятельство, что речь идет именно о классе деятельностей, то есть о таком образовании, которое имеет огромный объем своего содержания и столь же беспрецедентный диапазон и его качественных разновидностей, и степени их сложности. В этой связи можно дифференцировать два «вектора» качественной гетерогении данного класса деятельностей. Первая - это вертикальная гетерогения, вскрывающая глубокие принципиальные, то есть именно качественные, различия в степени сложности (ее пример как раз и представлен выше). Вторая - это горизонтальная гетерогения, вскрывающая фракт существования многих видов информационной деятельности, являющихся паритетными по сложности.

На основе этого возникает принципиальная проблема, являющаяся очень типичной и для исследования двух традиционных классов. Поскольку все множество конкретных разновидностей деятельности, образующих субъектно-информационный класс, невозможно раскрыть в одном, отдельно взятом исследовании, то необходимо избрать такую его экспликацию, которая была бы наиболее репрезентативной в плане основных характеристик и особенностей, феноменов и закономерностей. В свете этих критериев нет необходимости подробно обосновывать положение, согласно которому такой экспликацией (точнее, целым семейством принципиально сходных экспликаций) являются все те виды деятельности, которые базируются на компъютерных технологиях, а базовым средством труда в них выступает именно компьютерная техника. Именно их, по нашему мнению, и необходимо сделать предметом приоритетного исследования в плане изучения субъектно-информационного класса деятельности.

Таким образом, можно видеть, что исследование развития «мира деятельностей», приведя на современном этапе к необходимости дифференциации субъектно-информационного класса, ставит фундаментальную по своей теоретической и практической значимости проблему, которую можно обозначить как проблему «метакогнитивизм и компьютеризация», «метакогнитивизм и IT-технологии». Она включает в себя очень широкий спектр вопросов и проблем - как тех, которые уже отмечены выше, так и многих других, которые и должны выступить в качестве первоочередных. Подтверждением этого является и то, что исследования в данной области уже сейчас являются весьма актуальными и проводятся, в частности, в совре- 
Психологическая специфика профессиональной деятельности ...

менном метакогнитивизме, а также в смежных с ним областях (см. обзор В [11]).

\section{III}

Наиболее показательно, что именно при такой конкретизации предмета исследования сразу же - очень непосредственным и вполне естественным, даже необходимым, образом - выявляется обстоятельство наиболее принципиального плана и фрундаментального значения. В своем общем виде оно заключается в удивительном подобии - в принципиальном сходстве и в максимальной конгруэнтности самого “духа» метакогнитивизма (его сореры, предмета, специфики, задач, разделов и пр.) и основных качественных особенностей деятельности информационного характера, реализуемых на базе компьютерной техники. Поясним сказанное. Известно, что в структуре метакогнитивизма исторически сложились и являются в настоящее время основными две его «составляющие», два главных направления [12-14]. Первое имеет своим предметом исследование метакогнитивных процессов: это операционное направление, которое и закреплено в термине «метакогнитивизм». Второе направление имеет своим предметом знания, но особого типа - «знания о знаниях», то есть метазнания: это операндное направление, которое закреплено в понятии «психология метапознания». Фактически складывается ситуация, при которой обе эти основные «составляющие» метакогнитивизма «воплощаются» в сути информационной деятельности посредством компьютерной техники, но и сама техника выступает при этом в фрункции практически полного аналога и «первичных" процессов, и «первичных» знаний. Субъект же деятельности с необходимостью выступает при этом и как реализатор процессов по управлению этими «первичными» процессами», и как носитель, а также преобразователь знаний об этих «первичных» знаниях. Следовательно, такого рода субъектно-информационная деятельность может быть понята только как метакогнитивная. Между информацией (как предметом деятельности) и субъектом деятельности находится такое средство труда (компьютер), которое по самой соей сущности фактически выступает носителем целой системы процессов и системы знаний (базы данных). Они, однако, носят специфически когнитивный характер, причем взятые в их единстве. То метакогнитивное содержание, которое представлено в индивидуальной психике во внутреннем плане (в частности, в интрапсихической плоскости), в субъектно-информационных деятельностях оказывается представленным уже во внешнем плане, в том числе и в распределенном виде между самим субъектом и средством его труда. Можно видеть, что имеет место принципиально новая деятельностная реальность, которая никак не присуща двум традиционным классам и которая определяет качественное своеобразие третьего класса и его несводимость к первым двум. Эту реальность во всей ее полноте, сложности, а отчасти и необычности еще предстоит понять и осознать. 
Карпов А. В., Чемякина А. В.

Так, на основе выполненных нами исследований можно считать, что реальная, а не симплифицированная структурно-уровневая организация профессиональной деятельности существенно более сложна, нежели это полагается традиционно. Особенно ярко это проявляется именно в классе субъектно-субъектных видов (в частности, управленческой, педагогической, а также базирующейся на компьютерных технологиях); она образована не тремя, как это считается аксиоматичным, а пятъю основными уровнями. Она, наряду с известными, включает в себя еще и уровни, обозначенные понятиями метадеятельностного и инфрадеятельностного.

Действительно, и собственно эмпирические материалы, и методологические аргументы позволяют дифференцировать в общей структуре деятельности своеобразный качественно специфический уровень ее организации. Он локализуется между уровнем «автономной деятельности» и уровнем действий, заполняя собой огромный диапазон вариаций степени сложности ее организации между ними. Он характерен (и даже объективно необходим) для любой системы, поскольку раскрывает специфику функционирования ее основных субсистем (подсистем) и может быть обозначен в общем виде как субсистемный. По отношению же к деятельности как одной из сложнейших типов систем он адекватнее всего описывается понятием инфрадеятельностного уровня. Он находится под уровнем автономной деятельности (отсюда и название - инфрадеятельностный), но над уровнем отдельных действий. Он заполняет собой тот огромный диапазон качественно различных форм организации деятельности, которые располагаются между крайними полюсами ее сложности. Данный уровень соответствует не системе деятельности в целом и не ее компонентам (действиям). Следовательно, он соотносится не с мотивацией и целями как таковыми, а должен выделяться на основе критерия дифференциации уровней. Он характеризует деятельность не на системном и не на компонентном уровне ее реализации, а на субсистемном уровне.

Тем самым понятием (и реальностью), которому он наиболее полно, точно и строго соответствует, является понятие ситуации. Именно оно, как известно, не только является одним из ключевых теоретических понятий, необходимых для экспликации содержания деятельностей субъектно-информационного класса, но и вообще составляет суть и основной источник проблем и затруднений в ее практической реализации. Содержание деятельности и процесс преодоления проблемных ситуаций по отношению к деятельностям данного класса - это вообще во многом синодические сущности.

Наиболее важно то, что оба отмеченных выше уровня не только складываются и функционируют под решающим детерминационным влиянием собственно метакогнитивных детерминант, но во многом ими вообще образованы. Отсюда следует вывод наиболее принципиального плана: факторы метакогнитивного типа обусловливают трансформации того основного, 
Психологическая специфика профессиональной деятельности ...

что есть в деятельности - ее структурно-уровневой организации. Основные классы профессиональной деятельности качественно отличаются друг от друга в аспекте тех приоритетов и даже той совокупности уровней, которые характерны для каждого из них.

Возникает логичное предположение, согласно которому и при переходе от этих двух классов к третьему (субъектно-информационному) также будут иметь место качественные трансорормации структурно-уровневой организации деятельности. Однако то, какие именно трансформации будут возникать и каким закономерностям они подчиняются, пока остается неизвестным. Следовательно, фрормулируется важная в теоретическом плане задача выявления и интерпретации тех закономерностей, которые лежат в основе этих трансформаций. Другими словами, формулируется конкретная по содержанию, но общая и принципиальная по смыслу задача выявления того, каким образом в деятельностях субъектно-иноормационного класса трансформируется их общая структурно-уровневая организация? Какую роль в этом играют фракторы собственно метакогнитивного плана? То, что такие трансформации действительно имеют место, можно предположить с достаточно высокой степенью вероятности. Дело в том, что сама суть и главное предназначение компьютерных технологий в том и состоит, чтобы разгрузить субъекта от относительно более простых (а в перспективе и все более сложных) когнитивных функций. Однако столь же известно, что именно эти фрункции локализуются в структуре деятельности на ее относительно низших уровнях - операционном и частично действенном. Следовательно, на основе этого и возникает предположение, согласно которому в данном классе два указанных уровня могут существенно редуцироваться и фрактически исключаться из ее общей структуры (либо же качественно трансорормироваться и приобретать неизвестные пока фрормы). В этом плане модно констатировать и достаточно интересную закономерность. Если при переходе от субъект-объектного класса к субъект-субъектному классу в структуре деятельности дополнительные приоритеты и качественно новые фрункции обретали два отмеченных выше весьма специфических уровня, то при переходе к субъектно-информационному классу из нее фрактически исключаются также два уровня, но уже не относительно высшие, а относительно низиие.

\section{IV}

Наряду с этим, существует и еще один очень важный, но и более скрытый, имплицитный аспект исследования деятельностей субъектно-инорормационного класса, обращение к которому становится возможным именно с позиций исследования метакогнитивных детерминант ее организации. Так, выше мы уже подчеркивали удивительное подобие ЧЕМУ?, фрактически полную конгруэнтность данного класса и содержания самого метакогнитивизма. Однако такое подобие, доходящее до степени тождества, прослеживается и в еще одном плане. С одной стороны, хорошо известно, 
Карпов А. В., Чемякина А. В.

что важнейшим принципом всех компьютерных технологий является дифференциация оперативной памяти от всех иных типов и средств долговременной фиксации информации в компьютере (как аналога долговременной памяти). В связи с этим важнейшей компетенцией субъекта данного класса деятельностей выступает способность организовать обмен между ними, то есть фрактически взаимодействие актуальной и виртуалъной инорормации; грамотно и продуктивно специфицировать актуальную информацию и обеспечить максимальную адресацию к «информации по запросу». Опытный профессионал «чувствует», что именно достаточно принять к рассмотрению, а что можно «оставить за кадром», поскольку это в любой момент может быть актуализировано «информацией по запросу». Все это - неоспоримая и обыденная реальность информационных видов деятельностей. Однако столь же очевидно, что в основе всего этого лежат именно специфически метакогнитивные умения и средства, поскольку все они направлены именно на «базы данных» (на знания) и на процессы работы с ними (также инорормационные по своему содержанию). С другой стороны, трудно не видеть и того, что вся охарактеризованная «специфически компьютерная» ситуация, связанная с информационными переходами из актуальной формы презентации в потенциальную (и наоборот), с соотношением актуальных и виртуальных данных и пр., не просто удивительно похожа на ту, которая составляет суть основных информационных взаимодействий в самой психике - взаимодействий между осознаваемыл и неосознаваемъм уровнями ее репрезентации и переработки, - но и фрактически эквивалентна ей. Компьютер вновь «повторяет психику», но уже не в плане подобия структурной организации, а в плане базового принципа фрункциональной организации. Это - подобие его фрункциональной организации с организацией межуровневых взаимодействий двух основных фрорм презентации информации актуальной и виртуальной, «фигуровой» и «фоновой», осознаваемой и неосознаваемой. Все это приводит к фрормулировке еще более сложных задач, имеющих, однако, очевидную метакогнитивную составляющую. Более того, эти задачи с объективной необходимостью заставляют обратиться к одному из важных направлений именно метакогнитивизма - направлению, исследующему неосознаваемые средства и механизмы метакогнитивного плана [15].

Подчеркнем также, что при решении данной задачи в ее наиболее общем виде следует руководствоваться тем, что реальная онтология деятельности - ее действительное и полное бытие - эксплицируется, как известно, через так называемую "деятельностную фрормулу». Это триада базовых «составляющих» любой деятельности: ее субъект, объект и процесс их взаимодействия, то есть собственно деятельности, взятой в ее временно́й развертке. В данной связи очень показательным (и доказательным) является следующее обстоятельство. Каждый из этих трех компонентов выступает в качестве базовой метасистемы, оказывающей наибольшее специфициру- 
ющее влияние на метакогнитивную сферу по отношению соответственно к трем разным классам деятельности; поясним сказанное. Так, по отношению к субъект-объектным деятельностям метакогнитивная сфера обретает главные специфические особенности под влиянием тех особенностей и закономерностей, которыми характеризуется более общая по отношению к ней метасистема - индивидуальная психика самого субъекта деятельности. По отношению ко второму классу в качестве такой специфицирующей метасистемы выступает уже не «субъектный», а «объектный» член этой фрормулы, поскольку для него главную роль играют особенности и закономерности, обусловленные тем, что в его качестве выступают также субъекты, «другие люди» - социальные объектъ. Соответственно, и вся метакогнитивная сфера обретает ярко выраженную социоориентацию, а сам метакогнитивизм трансформируется в социальный метакогнитивизм. Данное обстоятельство подробно обосновано нами на материале исследования управленческой и педагогической деятельности. Однако есть основания полагать, что именно этой же общей и, по-видимому, фундаментальной особенности подчиняется и тот класс деятельности, который пока не был исследован в данном плане - субъектно-информационный. В нем метакогнитивная сорера, по всей вероятности, в наибольшей мере специфицируется еще одним, третьим (средним), членом этой «формулы»- самим процессом деятельности. Он, однако, должен быть взят также в специфическом и вполне конкретном проявлении, в аспекте тех средств и операционных механизмов, которыми и реализуется этот процесс. В их качестве как раз и выступает все то, что составляет содержание компьютерных технологий как таковых. При этом показательно (и доказательно), что ключевое из этих средств не только по существу, но даже этимологически иллюстрирует именно это обстоятельство: специфику процессу деятельностей субъектно-инорормационного класса придает в основном именно процессор как ключевой компонент всех компьютерных средств.

Наконец, на наш взгляд, нельзя оставить без внимания и еще один аспект рассматриваемой проблемы, являющийся к тому же достаточно сложным и дискуссионным, даже в известной степени «болезненным». Он состоит в том, что в адрес правомерности дифференциации субъектно-информационного класса как такового нередко высказываются возражения, смысл которых сводится к следующему. Очень многие (или даже практически большинство) существующие виды деятельности не только обязательно включают в качестве важнейшего компонента переработку информации, но и, вообще, во многом базируются на собственно информационном взаимодействии с объектом. В силу этого в значительной мере стирается грань между ними и субъектно-инорормационным классом, а само его выделение становится дискуссионным. Дело, однако, заключается не в этом непреложном фракте, а несколько в другом - гораздо более имплицитном обстоятельстве. Оно состоит в том, что в целом ряде деятельностей (кото- 
Карпов А. В., Чемякина А. В.

рые и образуют субъектно-информационный класс) имеет место не только и не просто «инорормационное взаимодействие» или «переработка субъектом инфрормации», а качественно иная ее организация. Главной особенностью этой организации является то, что информация выступает не только в качестве основы деятельности. Сама деятельность в целом и средства ее реализации («орудия труда») в особенности таковы, что они эту информацию активно преобразуют, то есть сами ее перерабатывают, беря тем самым на себя часть фрункций, которые в иных классах деятельности являются исключительной прерогативой субъекта. Происходит «отчуждение» ряда исходно субъектных задач и функций, их перенос на реализацию средствами труда (если использовать традиционную терминологию), а сами эти средства выступают не только как пассивные орудия, полностью регулируемые субъектом, но и как в известном смысле активные ее реализаторы. Налицо, таким образом, полная аналогия данной ключевой особенности с той, которая является важнейшей и для дифференциации субъект-объектного класса деятельности. Как показано в наших исследованиях, он тем и отличается от всех иных классов, в особенности от профессией типа «человек-человек», что его предметом является не просто человек, а именно человек действующий, то есть субъект какой-либо своей собственной деятельности. Им является личность, сама активно осуществляющая какую-либо деятельность, сама являющаяся субъектом своей собственной деятельности.

\section{$\mathrm{V}$}

На основе вышеизложенного можно сделать следующее заключение. С одной стороны, дополнение теории деятельности изучением субъект-субъектных видов, а также введение в ее концептуальный состав самой дифрференциации деятельности на объектно-ориентированные и субъектно-ориентированные является очень логичным и естественным, объективно необходимым и понятным. Однако, с другой стороны, уже сама эта дифрференциация, означающая введение в нее еще одного класса изучаемой реальности, создает своеобразный прецедент, смысл которого заключается в том что, если действительно существует еще один класс, то возможно существование и других также дополнительных, новых и качественно отличных, классов, каковым и является субъектно-информационный класс.

Наконец, в данной связи необходимо учитывать и еще одно очень значимое и носящее совершенно объективный характер обстоятельство. Оно состоит в том, что наиболее общей своего рода «магистральной» тенденцией развития типов и форм организации профессиональной деятельности как раз и является все более широкое распространение деятельностей именно субъектно-информационного характера. Решающим шагом в этом направлении является, разумеется, крупнейший технологический «прорыв», приведший к беспрецедентному распространению компьютерных технологий. В целом это переход общества в так называемую «IT-эпоху», дополнение всех сорер жизни общества новым, «пятым», измерением реальности, 
метафрорически обозначаемым, как известно, понятием «е-измерения» [1]. И именно субъектно-информационные виды деятельности со всей остротой и очевидностью обнаруживают явную недостаточность традиционных вариантов психологической теории, а также декомпозиции самой деятельности на основные уровни. Вместе с тем именно их изучение одновременно позволяет наметить и пути дальнейшего развития этой теории, одной из основных среди которых является необходимость ее синтеза с теми данными, которыми располагает современный метакогнитивизм. На основе именно этого синтеза окажется возможным становление нового направления психологических исследований - метакогнитивной психологии деятельности.

\section{Ссылки}

1. Абдеев Р. Ф. Философия информационной цивилизации. М.: Владос, 1994. $336 \mathrm{c}$.

2. Карпов А. В., Леньков С. Л. Структурно-функциональное строение профрессиональной деятельности информационного характера. Тверь: ТГУ, 2006. 448 с.

3. Климов Е. А. Психология профессионала. Воронеж: МПСИ, 1996. 400 с.

4. Роберт И. В. Современные информационные технологии в образовании: дидактические проблемы, перспективы использования. М.: Школа-Пресс, 2004. 210 с.

5. Kluwe R. H. Cognitive Knowledge and executive control: Metacognition // Animal mind-human mind / D. R. Griffin (Ed.). N. Y.: Springer-Verlag. 1982. P. 201-204.

6. Карпов А. В. Психология сознания. М.: РАО, 2015. 1080 с.

7. Карпов А. В., Карпов А. А. Методологические основы психологии образовательной деятельности. Т. 2: Когнитивное обеспечение. М.: РАО, 2018. 580 с.

8. Зинченко В. П. Сознание и творческий акт. М.: ВШЭ, 2010.582 с.

9. Леньков С. Л. Субъектно-информационный подход к психологическим исследованиям. Тверь: Тверской гос. ун-т. URL: https://www.elibrary.ru/item. asp?id=42979173 ( дата обращения: 30 апреля 2021).

10. Трудности и перспективы цифровой трансформации образования / под ред. А. Ю. Уварова, И. Д. Фрумина; М.:ВШЭ, 2019. 343 с.

11. Cognition, Metacognition, and Culture in STEM Education: Learning, Teaching and Assess-ment (Innovations in Science Education and Technology, 24) Softcover reprint of the original 1st ed. / by Y. Dori, Z. Mevarech, D. Baker. Springer, 2018. 150 p.

12. Karpov A. V., Karpov A. A. The interconnection of learning ability and the organization of metacognitive processes and traits of personality // Psychology in Russia: State of the Art. 2017, Issue 1. Vol. 10. P. 67-79.

13. Metacognition: Cognitive and Social Dimensions / Ed. By V. Yzerbyt [et al.] SAGE Publica-tions, 2002. 253 p.

14. Pilot A Metacognition in Higher Education. Twente University Press, 2021. 154 p.

15. Koriat A. Conscious and Unconscious Metacognition: A Rejoinder // Consciousness and Cognition. 2000. 9. P.193-202. 